\title{
Utilisation of the Coherence Analysis in Acoustic Diagnostics of Internal Combustion Engines
}

\author{
Zbigniew DĄBROWSKI, Jacek DZIURDŹ, Dorota GÓRNICKA \\ Institute of Machine Design Fundamentals \\ Warsaw University of Technology \\ Narbutta 84, 02-524 Warszawa, Poland, e-mail: zdabrow@simr.pw.edu.pl
}

(received March 5, 2017; accepted April 10, 2017)

\begin{abstract}
The leading thought of the present text is that the internal combustion engine noise - regardless of all possible inconveniences - is a good information carrier that allows for the explicit diagnostics of such subtle defect as the valve face decrement which is so small that it is in practice 'unnoticeable' by the standard diagnostic-controlling system of a passenger car. However, such defect at its further propagation can be much more dangerous. Thus, diagnosing its initial state is worth trying. This study is a continuation of investigations where efforts were undertaken to perform the efficient diagnostics on the bases of observations and analyses of mechanical vibrations of the engine head.
\end{abstract}

Keywords: acoustic diagnostics; internal combustion engines; mechanical defects.

\section{Introduction}

An acoustic diagnostics of temporary defects of internal combustion engines was the first form of noninvasive diagnostics applied in times when the only interpretation of observations was the subjective impression of the well trained and experienced mechanic. It would seem that a huge development of measuring techniques, especially numerical transformations of signals, will make the 'organoleptic' diagnostics a reliable tool of instrumental investigations. However, this did not happen for certain reasons. Firstly, modern internal combustion engines are much better attenuated and changes of their acoustic signals are more difficult to observe. Secondly, a relatively lower noise level is masked - to a much higher degree than years ago by noises of other mechanisms (including the power transmission system), especially by aerodynamic and roadway noises. Finally, changes in noise that are accompanying engine abnormal operations are so low in energy that they do not measurably increase the overall level of sound energy in a relatively wide measuring band. Thus, the natural conclusion was to look for the satisfactorily narrow band ('spectral line'), sensitive to the state of the selected element or set. Unfortunately the acoustic signal transformation - source-receiver - is a non-linear transformation and in consequence changes of amplitudes as well as of frequencies occur.
Thus, the so-called characteristic band, in which the observed amplitude changes would be proportional to the development of individual defects, does not exist. Due to this reason the only defects which can be diagnosed relatively easily, on the basis of the engine noise analysis, are bolt joints or crank bearing clearances manifesting themselves by characteristic "slapping", i.e. impulse noises synchronous with the engine rotational speed. In the current diagnostic practice various kinds of acoustic probes and stethoscopes for locating the place of occurrence of the 'abnormal' noise source (probably related to a defect) are applied. Supporters of utilising vibroacoustic methods, in the detailed diagnostics, consider vibration symptoms much more often (Komorska, Puchalski, 2015; Konieczny et al., 2015). In this last case two techniques can be observed. The first one, generally considered as traditional, is based on creating the model (more or less accurate) of the vibroacoustic energy propagation, its identification and the generation of the expected symptom being the initial process change (DĄBROWSKI, 1992; Herrin, 2019; Zhou, Crocker, 2010). The second technique, more and more generally applied, utilises the huge calculation potential of modern computers and is based on collecting large numbers of recording and searching, by means of advanced mathematical techniques, for small changes which can be related to the object state change (defect development). Ge- 
netic, evolutional algorithms are especially popular. It is worth to mention here the modern techniques utilising the chaos effect (BATKO et al., 2008) or the fractal analysis (PUCHALSKI, 2013).

The leading thought of the present text is that the internal combustion engine noise - regardless of all possible inconveniences - is a good information carrier that allows for the explicit diagnostics of such subtle defect as the valve face decrement which is so small that it is in practice 'unnoticeable' by the standard diagnostic-controlling system of a passenger car. However, such defect at its further propagation can be much more dangerous. Thus, diagnosing its initial state is worth trying. This study is a continuation of investigations given in (GóRNICKA, 2016), where efforts were undertaken to perform the efficient diagnostics on the bases of observations and analyses of mechanical vibrations of the engine head.

\section{Research experiment}

The investigated object constituted the four-inline 1.2 engine of spark ignition, controlled by means of the electronic injection-ignition SPI (Single Point Injection) system. The unit of a significant mileage $(400000 \mathrm{~km})$ was selected for tests. Isolating the symptom from the signal generated by the vehicle of so advanced operational state, allowed to confirm the universality of concluding the valve defect, regardless of the wearing state of the remaining systems.

Noise measurements under the bonnet were carried out by means of the measuring microphone B\&K 4189A21, mounted to the bonnet using an elastic element. This microphone was practically free of the mechanical vibrations in the observed band. The head vibrations were measured by means of the piezoelectric accelerometer B\&K 4393. The multi-channel measuring cassette B\&K PULSE 3560 E (Fig. 1) was used for recording signals.

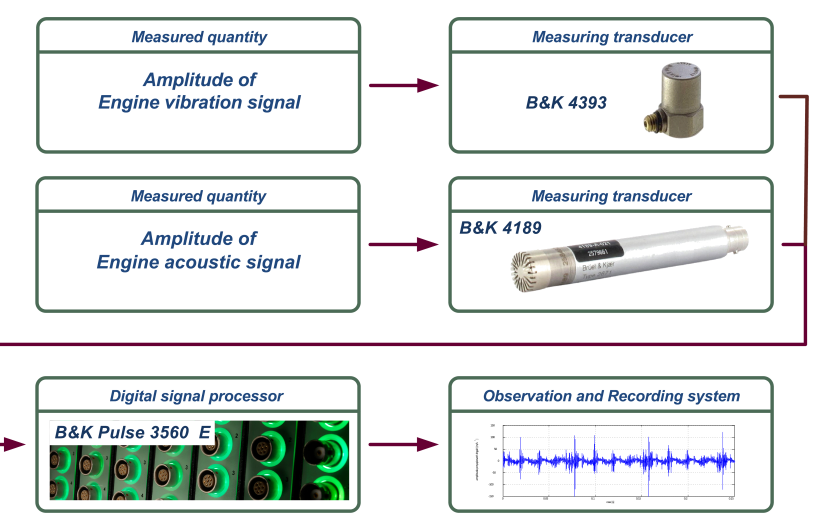

Fig. 1. Scheme of the recording system.

Since the measure sought should be able to serve as support for existing "on-line" diagnostic systems, it is necessary to select the symptom from the signal generated by engine operating in real driving conditions. Thus the signal recording was performed under the road conditions, while maintaining constant values of rotational speeds (1500, 2000, 3000, $4000 \mathrm{rot} / \mathrm{min})$ for five gears (with an accuracy resulting from the control system possibility).

The following technical conditions were tested:

- initial condition ('operational'),

- I defect stage of an exhaust valve,

- II defect stage of an exhaust valve, corresponding to the defect development,

- condition after exchanging the defected valve for the new one, without its preliminary matching (running in).

The valve defect was simulated by making the incision in the valve face $3 \mathrm{~mm}$ deep - which corresponds to the I stage of defect (defected surface $\sim 5.2 \mathrm{~mm}^{2}$ ) and $6 \mathrm{~mm}$ deep as the II stage of defect (defected surface $\sim 20.8 \mathrm{~mm}^{2}$ ). The way of making these incisions is shown in Fig. 2.
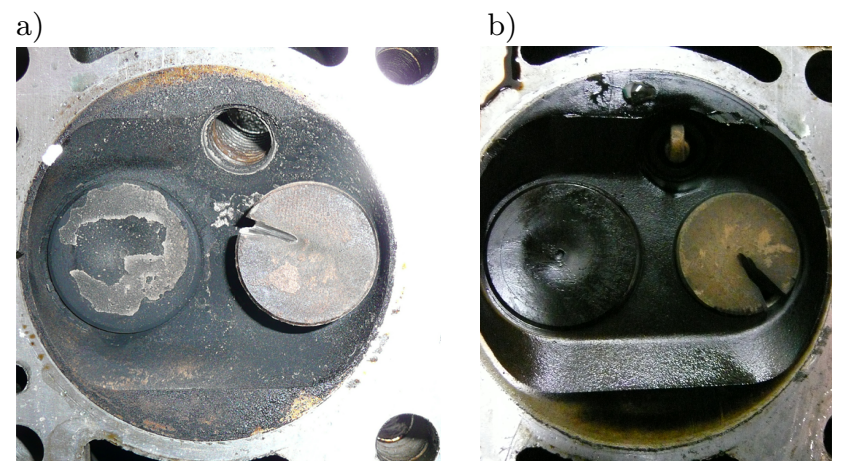

Fig. 2. Simulated defect of the exhaust valve: a) the first stage of defect (incision $3 \mathrm{~mm}$ deep), b) the second stage of defect (incision $6 \mathrm{~mm}$ deep).

\section{Methodology of the analysis}

Applying elements of the coherence analysis in the diagnostics task requires explanations (BATKO et al., 2006; 2008; Dziurdź, 2013; DąBrowski, Dziurdź, 2016). According to the generally known definition (Randall, 1987; Bendat, Piersol, 2010) the ordinary coherence function is a probabilistic characteristics of the system having one input and one output. The spectral transmittance of the described system can be determined in two ways:

$$
\begin{aligned}
& H^{(1)}(i, \omega)=\frac{G_{x y}(i, \omega)}{G_{x x}(i, \omega)}, \\
& H^{(2)}(i, \omega)=\frac{G_{y y}(i, \omega)}{G_{y x}(i, \omega)},
\end{aligned}
$$

where $G_{x x}$ is the power spectral density of input, $G_{y y}$ is the power spectral density of output, $G_{x y}$ is the 
cross spectral density from input to output, $G_{y x}$ is the cross spectral density from output to input, $H^{(1)}$ and $H^{(2)}$ are spectral transmittances determined in the first and second way.

For the undisturbed linear system: $H^{(1)}=H^{(2)}$. When the system is non-linear or when the disturbance occurs either in input or in output: $H^{(1)} \neq H^{(2)}$. Thus, the coherence function:

$$
\begin{gathered}
\gamma^{2}(\omega)=\frac{H^{(1)}}{H^{(2)}}=\frac{\left|G_{x y}\right|^{2}}{G_{x x} G_{y y}}<1 \\
\text { and generally } \quad 0 \leq \gamma^{2} \leq 1 .
\end{gathered}
$$

Let us assume now that the observed noise is a random multidimensional process

$$
Y=\{Y(t, \theta, n, r, \Omega)\}
$$

where $t$ is the observation time, $\theta$ is the evolutional variable of a time or defect parameter dimension (state variable), $n$ is the machine discriminant, $r$ is the location of the measuring perceptor, $\Omega$ is the engine rotational speed (the gear position, i.e., the relation of the rotational speed to the vehicle velocity $-\Omega / v$ should be often taken into account).

The observed signal is the output of the system whose input is relatively difficult to observe and - in practice - impossible to measure direct. The 'physical' source of the observed processes is the combustion process in the cylinder which generates the noise observable by means of the engine structure, exhaust system, and dynamics of mechanisms (mainly pistoncrankshaft system).

Thus, to be able - in the diagnostics process to conclude a defect on the bases of transmittance changes, their sources should be identified (eventually defined) on the basis of the selected intermediate observation.

Let us discuss the simple system of two outputs, presented in Fig. 3.

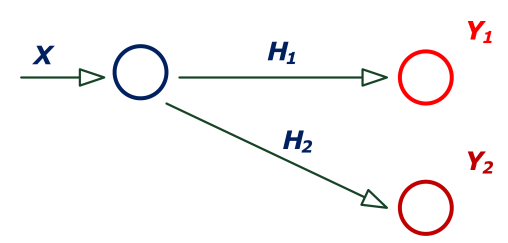

Fig. 3. Simple system of two outputs.

General relations are valid for this system:

$$
\begin{aligned}
& Y_{1}=X \cdot H_{1} \\
& Y_{2}=X \cdot H_{2}
\end{aligned} \quad \Rightarrow \quad Y_{1} \cdot H_{1}^{-1} \cdot H_{2}=Y_{2},
$$

from which it results that instead of observing the real source we can observe another output. Of course, in order to be able to fully use Eq. (4) the transmittance
$H_{1}$ should be known. However, let us note that when the transformation is reduced to the modules analysis:

$$
G_{x x} \cdot|H|^{2}=G_{y y}
$$

the amplification coefficient $|H|^{2}$ has the meaning of the scale function. From this the following statement directly results:

$x_{1}$ and $x_{2}$ are in a linear relation with each other:

$$
x_{2}=L x_{1} \Rightarrow \hat{\omega}_{2} x_{2}\left(\omega_{i}\right)=k x_{1}\left(\omega_{i}\right)
$$

then

$$
\hat{\omega}^{2} \gamma^{2}=\frac{\left|G_{x_{2} y}\right|^{2}}{G_{x x_{2}} \cdot G_{y y}}=\frac{\left|G_{x_{1} y} \cdot k\right|^{2}}{G_{x x_{1}} k^{2} \cdot G_{y y}} .
$$

This means that the linear scaling of the source spectral density does not change the coherence function value.

This statement is of a key meaning for applying the coherence function as diagnostically useful process characteristics. In particular, it states that there is no need of investigating the relation between the 'real' source and the observed process treated as the output, and that investigating of the coherence function between the scaled input - in practice a certain standard - is satisfactory. Let us return now to the diagnostics task. In accordance with the above, we are expecting that the defect occurrence will modify the observed signal in a non-linear way. Thus, the observable change of the spectrum structure will occur in certain bands. Utilising filtering properties of the coherence function we will consider this change as the disturbance $z(t)$ of the system 'output' (Fig. 4 in which $x(t)$ is the input, $v(t)$ is the output without disturbances, $z(t)$ is the disturbance process, $y(t)$ is the observed output), which allows to apply the dependence (Bendat, Piersol, 2010; DĄBrowski, Dziurdź, 2016):

$$
\begin{aligned}
& H^{(1)}=\frac{G_{x v}}{G_{x x}}=\frac{G_{x y}}{G_{x x}}=H_{x y}, \\
& H^{(2)}=\frac{G_{y y}}{G_{y x}}=\left(1+\frac{G_{z z}}{G_{y y}-G_{z z}}\right) H_{x y}, \\
& \gamma_{x y}^{2}=\frac{H^{(1)}}{H^{(2)}} \Rightarrow\left(1-\gamma^{2}\right) G_{y y}=G_{z z} . \\
& \stackrel{\boldsymbol{x}}{\triangleright}
\end{aligned}
$$

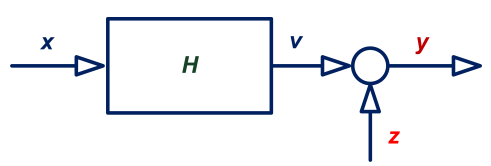

Fig. 4. System with the disturbed output, caused by a defect.

In effect, we are filtering out - from the signal - a certain non-linear disturbance, being the defect symptom. However, such an operation requires satisfying two conditions. Firstly, on the basis of the experimental results a possibly narrow frequency interval in which the analysis will be performed should be 
a)

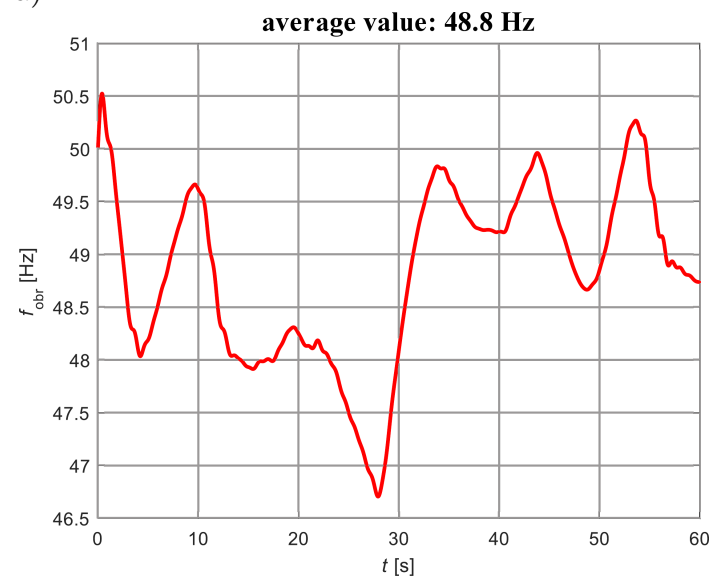

b)

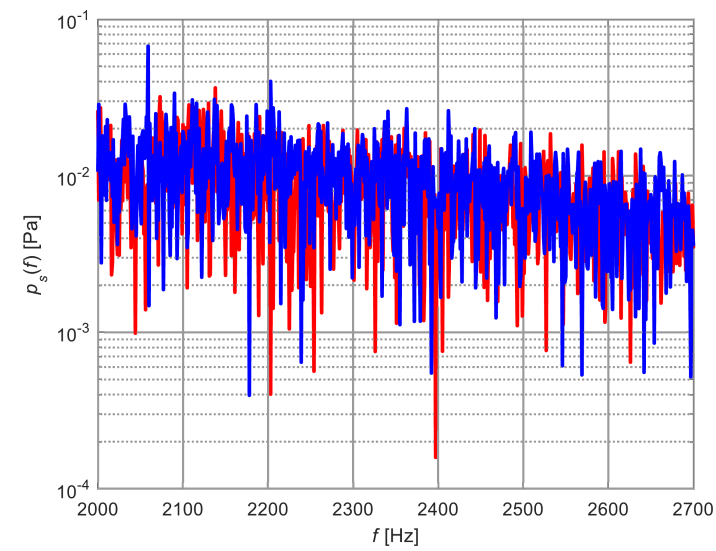

Fig. 5. Effect of fluctuations of the 'theoretically' constant rotational speed: a) fluctuation of the engine rotational speed, b) power spectral density of two realisations.

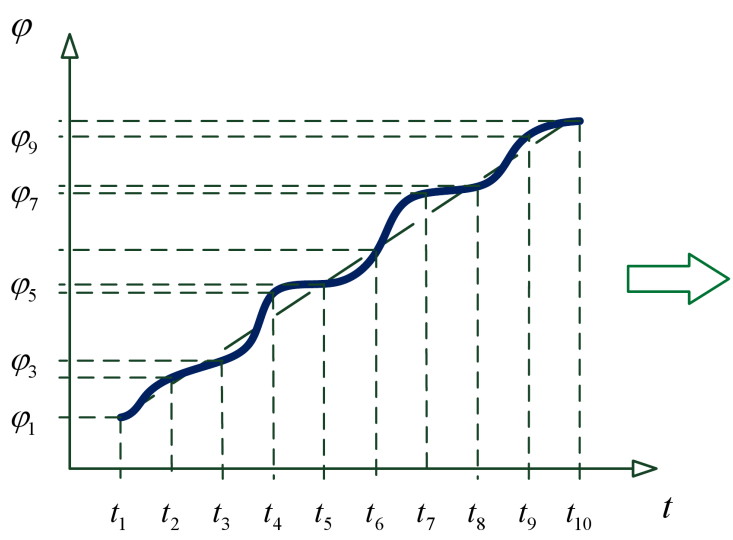

$\Delta t=$ const

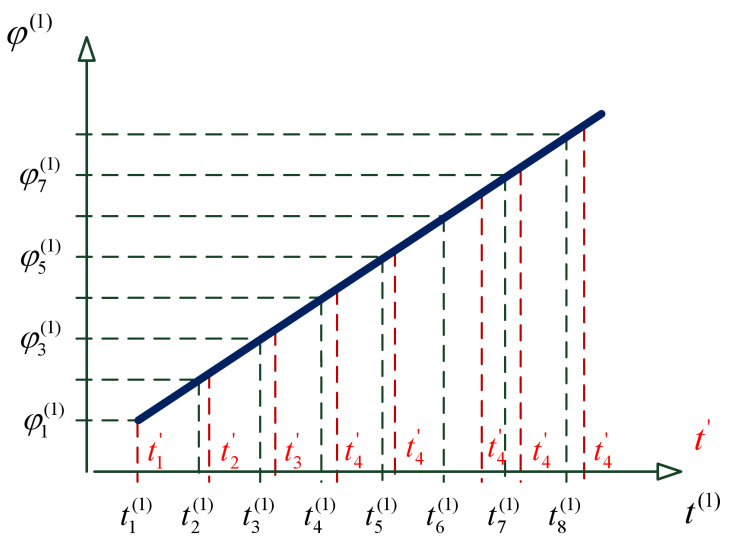

$\Delta t^{(1)}\left(\varphi^{(1)}\right)=$ const

Fig. 6. Effect of the signal resampling warranting to satisfy the condition: $\Delta \varphi=$ constant.

determined. Thus, the observed signal should be assumed as:

$$
\Im \bar{Y}=\underset{\omega}{S} \Im \underset{t}{S} Y(t, \theta, r, n, \Omega)
$$

where $\underset{t}{S}$ is the selection operator in the time domain, defining the recording parameters, $S$ is the selection operator in the frequency domain (filtration), defining the band selection, $\Im$ is the Fourier transform.

Secondly, the identical observation conditions should be maintained all the time, which means:

$$
r=\text { const }, \quad n=\text { const }, \quad \Omega=\text { const. }
$$

The third of this conditions is very difficult to satisfy, since even when the best speedometers (tempomates) are used, the rotational engine speed is fluctuating, which is shown in Fig. 5. Thus, providing the proper synchronisation is necessary. Several methods can be applied. The authors of the paper are consequently using the signal resampling technique proposed by (DZIURDŹ, 2010; DĄBROWSKI, DZIURDŹ, 2016 ), reduced to the change of the time scale into the angle of rotation scale (Fig. 6).

\section{Analysis of the results}

Noise spectra for various states (after synchronisation of the rotational speed) are presented in Fig. 7. It is obvious that the direct spectrum analysis does not allow for performing diagnostics.

In accordance with Eq. (8) the selection was performed within the band: $2100<f<2700 \mathrm{~Hz}$. The spectrum of the recorded mechanical vibrations of the head in the selected band, after removing noises and rescaling into the same level bands spectrum (according to Eq. (6) Fig. 8) and after filtering out non-linear disturbances (according to Eq. (7)), was assumed as the standard (apparent source).

Residual spectra (incoherent signal parts) for the maintenance state and for two stages of the valve defects are shown in Fig. 9. 

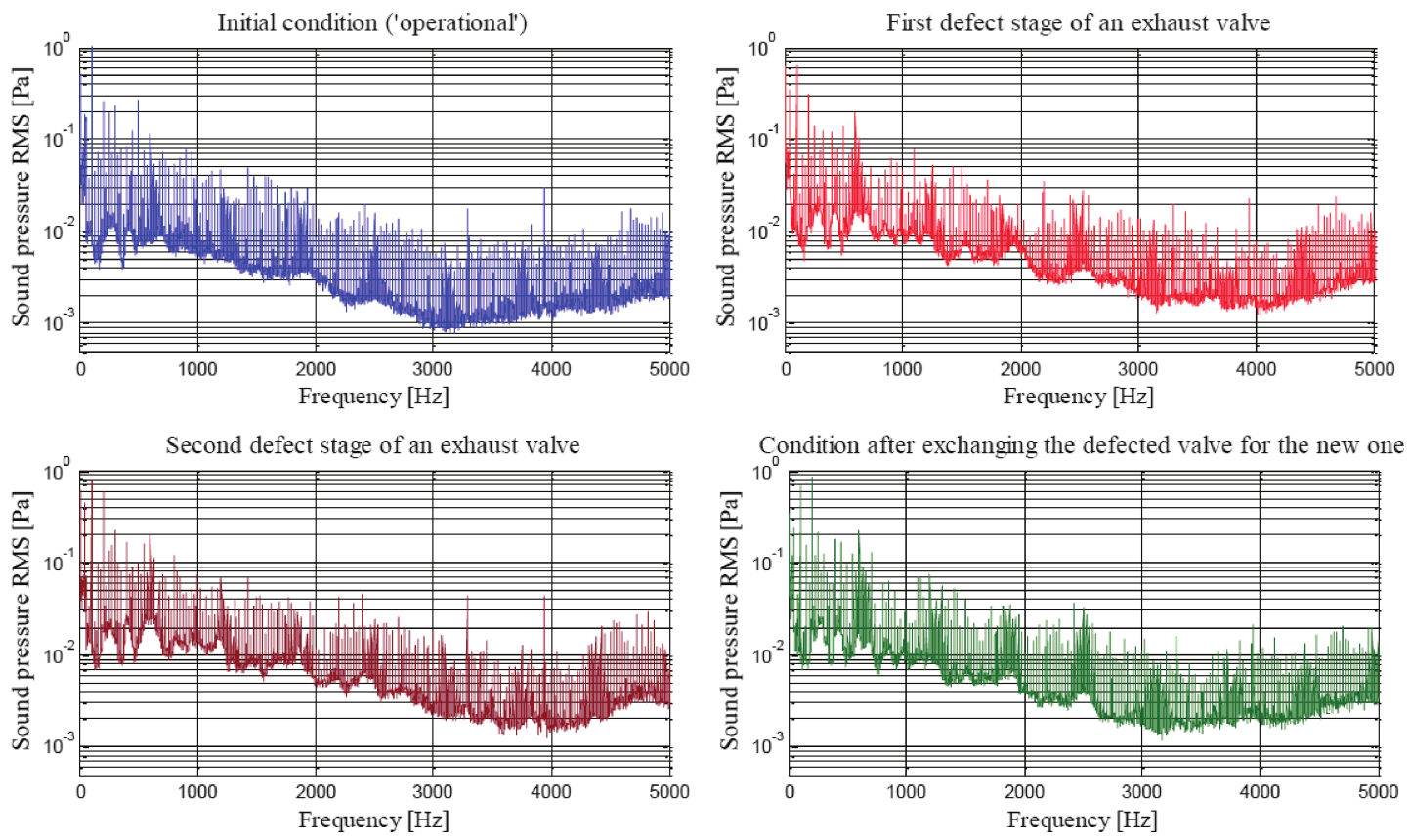

Fig. 7. Power spectral density of the signals recorded for various states.
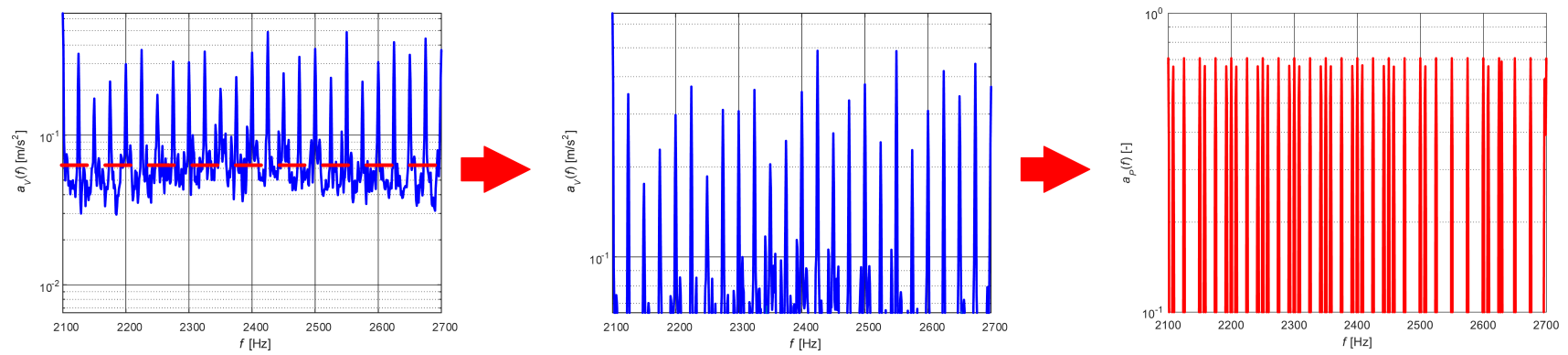

Fig. 8. Schematic presentation of the standard formation (apparent input).
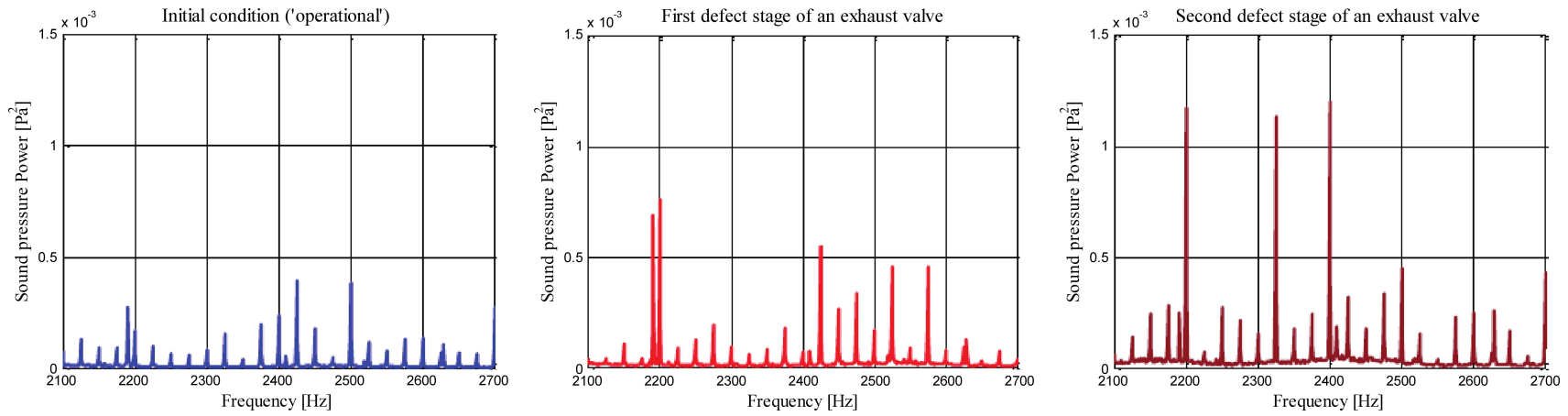

Fig. 9. Residual spectra in the selected band for various states.

The simple measure „Total” from the selected (in the described above way) residual spectra constitutes the monotonic measure of the defect (Fig. 10).

This last figure is a convincing evidence of the proposed methodology's efficiency. As a formality it would be right to check the behavior of the new valve in this way of the state assessing. The result, at the first glance, seems surprising. The residual spectrum ob- tained after exchanging the defected valve with the new one is shown in Fig. 11.

From the interpretation of the proposed measure it results that the new valve behaves as the defected element (!). However, the explanation of this effect is quite simple. The valve installed into the old, rough valve seat is thus less tight than the valve with a defected face (this can be easily confirmed by 


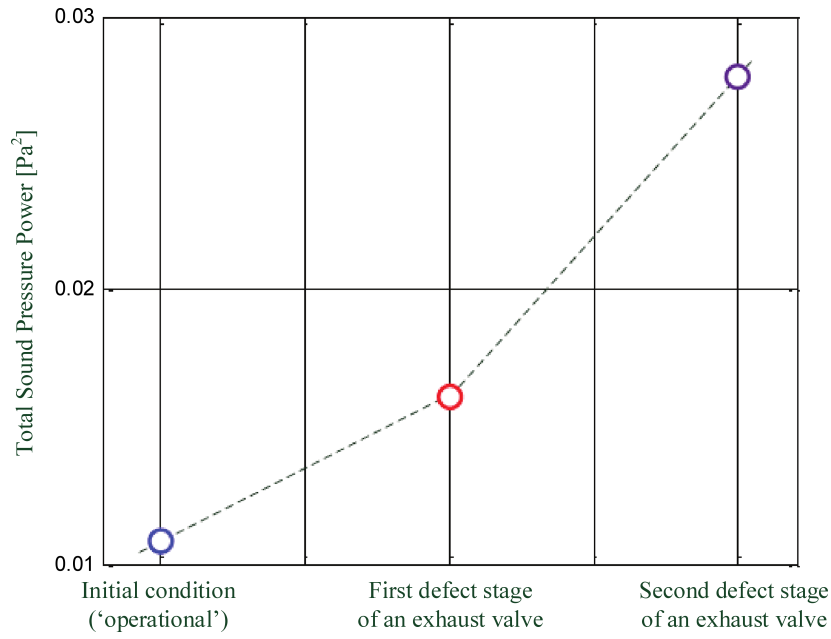

Fig. 10. Simple measure of the defect.

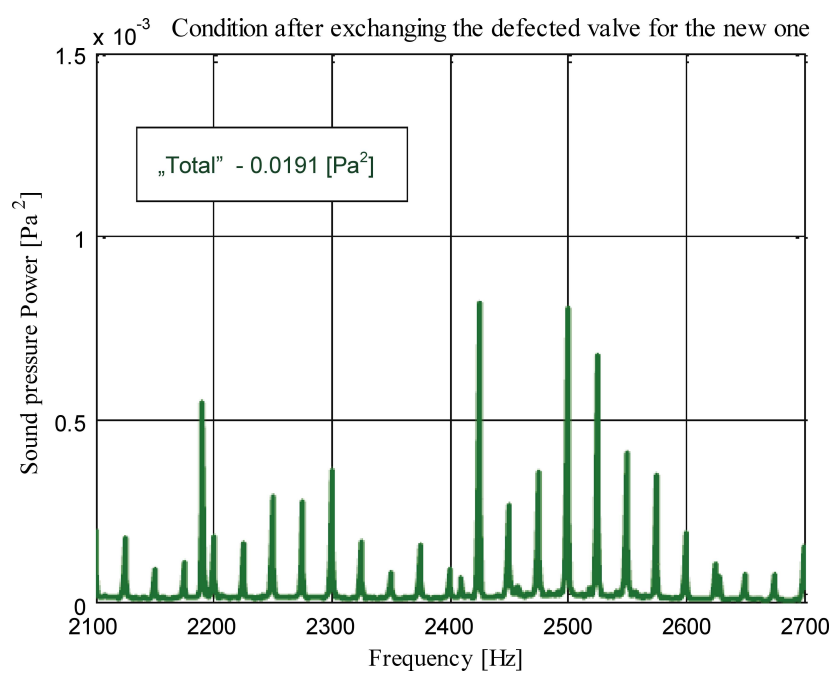

Fig. 11. Effect of not correct installation of the new valve.

checking the mean pressure in the cylinder). It was found in further tests that as far as the after-repairs mileage increases the applied measure decreases fast. However, according to the authors, it is much better to perform the correct repair.

\section{Conclusions}

The obtained results allow to draw two basic conclusions.

1) Noises under the passenger car bonnet, in the vehicle of a high wearing degree and going on various roads, are measured under very inconvenient conditions. However, regardless of many disturbances, it is possible to find information concerning small mechanical defects such as a subtle defect of the exhaust valve face.

2) Generalisation of the coherence analysis into investigations of the input $\leftrightarrow$ output relation for the input - understood not as the real source but as a certain apparently assumed standard and for outputs differentiated not by a spatial variable (another measuring point) but by the object state variable, can constitute the efficient tool of finding diagnostics information. This confirms the thesis that the non-linear disturbance of the system dynamic answer increases due to the defect propagation.

\section{References}

1. Batko W., Dabrowski Z., Kicinski J. (2008), Nonlinear Effects in Technical Diagnostics, Publishing and Printing House of the Institute for Sustainable Technologies - NRI, Radom.

2. Batko W., Dabrowski Z., Engel Z., Kiciński J., Weyna S. (2006), Modern Methods of Research Vibroacoustic Processes [in Polish: Nowoczesne metody badania procesów wibroakustycznych], Publishing and Printing House of the Institute for Sustainable Technologies - NRI, Radom.

3. Bendat J.S., Piersol A.G. (2010), Random data: analysis and measurement Procedures, 4th ed., Wiley, New York.

4. DĄBrowski Z., DziUrdŹ J. (2016), Simultaneous analysis of noise and vibration of machines in vibroacoustic diagnostics, Archives of Acoustics, 41, 4, 783789 .

5. DAzBrowski Z. (1992), The evaluation of the vibroacoustic activity for the needs of constructing and use of machines, Machine Dynamics Problems, Vol. 4, Warsaw.

6. Dziurdz J. (2010), Transformation of Nonstationary Signals into "Pseudostationary" Signals for the Needs of Vehicle Diagnostics, Acta Physica Polonica A, 118, 1, 49-53.

7. DzIURDź J. (2013), Analysis of nonlinear phenomena in diagnosing of the vehicle drive systems [in Polish: Analiza zjawisk nieliniowych $w$ diagnozowaniu uktadów napędowych pojazdów], Publishing and Printing House of the Institute for Sustainable Technologies - NRI, Radom.

8. GóRNICKA D. (2016), Utilising the vibration signal in diagnosing defects of valves in internal combustion engines [in Polish: Wykorzystanie sygnatu drganiowego w diagnozowaniu uszkodzeń zaworów silników spalinowych], PhD Dissertation, Warsaw University of Technology.

9. Herrin D.W., Liu J., Martinus F., Kato D.J., Cheah S. (2010), Prediction of sound pressure in the far field using the inverse boundary element method, Noise Control Engineering Journal,58, 1, 74-82.

10. Komorska I., Puchalski A. (2013), A Vibroacoustic diagnostic system as an element improving road 
transport safety, International Journal of Occupational Safety and Ergonomics, 19, 3, 371-385.

11. Konieczny L., Burdzik R., Warczek J., Czech P., Wojnar G., Mlynczak J. (2015), Determination of the effect of tire stiffness on wheel accelerations by the forced vibration test method, Journal of Vibroengineering, 17, 8, 4469-4477.

12. Puchalski A. (2013), Selected problems of diagnostics of vehicles mechanotronic systems [in Polish:
Wybrane problemy diagnozowania ukladów mechatronicznych pojazdów], Publishing and Printing House of the Institute for Sustainable Technologies - NRI, Radom.

13. Randall R.B. (1987), Frequency analysis, Brüel \& Kjær.

14. Zhou R., Crocker M.J. (2010), Boundary element analyses for sound transmission loss of panels, Journal of the Acoustical Society of America, 127, 2, 829-840. 